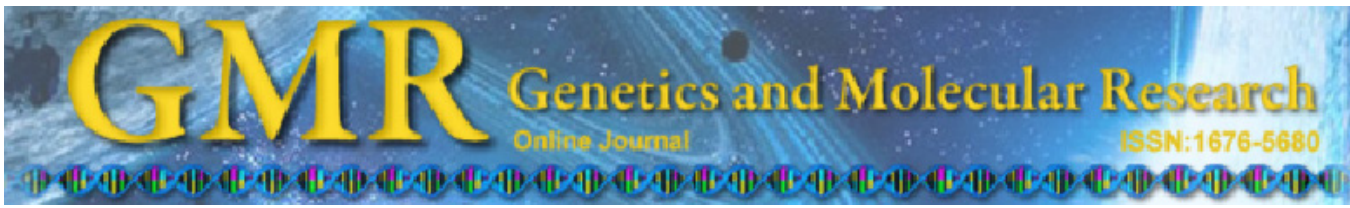

\title{
Inheritance of kernel row number, a multicategorical threshold trait of maize ears
}

\author{
F.H.R.B. Toledo ${ }^{1}$, M.A.P. Ramalho ${ }^{2}$, G.B. Abreu ${ }^{3}$ and J.C. de Souza \\ ${ }^{1}$ Programa de Pós-Graduação em Genética e Melhoramento de Plantas, \\ Escola Superior de Agricultura "Luiz de Queiroz", \\ Universidade de São Paulo, Piracicaba, SP, Brasil \\ ${ }^{2}$ Departamento de Biologia, Universidade Federal de Lavras, \\ Lavras, MG, Brasil \\ ${ }^{3}$ Programa de Pós-Graduação em Genética e Melhoramento de Plantas, \\ Universidade Federal de Lavras, Lavras, MG, Brasil \\ Corresponding author: F.H.R.B. Toledo \\ E-mail: fernandohtoledo@gmail.com
}

Genet. Mol. Res. 10 (3): 2133-2139 (2011)

Received January 3, 2011

Accepted June 27, 2011

Published September 21, 2011

DOI http://dx.doi.org/10.4238/vol10-3gmr1114

\begin{abstract}
Information about the inheritance of threshold traits is scarce, especially in plants. We examined the genetic control of kernel row number in maize (Zea mays). Knowledge of this inheritance is especially important because it is a primary component of grain yield. This trait has a discontinuous distribution. Characters like these are conceptualized as threshold traits. Crosses were made between the inbred line Geneze 3 (G3) with many kernel rows and the inbreds Argentino IV (A4) and Dente de Cravo (DC), with fewer kernel rows. The $\mathrm{F}_{1}$ and $\mathrm{F}_{2}$ generations and the backcrosses $\mathrm{BC}_{11}$ and $\mathrm{BC}_{21}$ were obtained for the combinations G3 x A4 and G3 x DC. These populations were evaluated under field conditions, and the kernel row number was determined by direct counting of approximately 14, 140 and 75 ears for the $F_{1}, F_{2}$ and backcrosses, respectively. Genetic control was determined through estimates of generation means and variance analysis and was also performed by Wright's method for threshold traits. It was found that genetic control is predominantly due to additive alleles. The component $a$, was greater than zero, additive variance was
\end{abstract}


positive and the variance of dominance did not differ from zero. In the $\mathrm{F}_{2}$ generation, the range of the kernel row number was 10 to 28 in G3 x A4, while in G3 x DC it was 12 to 26. Inheritance of the number of kernel rows, estimated by the two methods, gave similar results. This correspondence is due to adjusting of the data to the normal distribution.

Key words: Crop improvement; Threshold traits; Kernel row number

\section{INTRODUCTION}

There are broad researchs on methodologies for analyzing the inheritance of quantitative traits (Wricke and Weber, 1986; Bernardo, 2002). However, there are some characters that are controlled by many genes and influenced by the environment, and as their expression takes place in a discreet manner, they are conceptualized as threshold traits (Lynch and Walsh, 1997). One of the characters that fall in that category is kernel row number on maize ears. This number is always in pairs but there is wide variation. The first research on the genetic control of this character was performed by East (1910), one of the pioneers of quantitative genetics, where new information about this character was only found after more than 90 years (Ross et al., 2006; Srdic et al., 2007).

Wright $(1934 a, b)$ was the first scientist to develop methodologies for the study of threshold characters. He was interested in the inheritance of the number of digits in guinea pigs. The basis of the study of threshold characters is that even phenotypic observations show discontinuity. There is a normal distribution on an underlying scale, and thus, these characters are essentially quantitative, but the thresholds impose a discontinuity in phenotypic expression (Falconer and Mackay, 1995).

The particularity of this character is evident by the meager amount of research on this topic, with few of them relating to plants (Wright, 1943; Frank and Hallauer, 1997). Frank and Hallauer (1997) studied the genetic control of twin-ears in maize, which is a threshold trait, but these authors interpreted the segregations obtained using the methodology usually employed for quantitative traits. Considering the number of threshold traits in plants, it is important to check whether you need to use the specific method of Wright (1934a,b), which is widely used in studies of animal characters in contrast to least mean squares. Therefore, the aim of this study was to obtain information about the inheritance of kernel row number and to verify if the genetic parameters estimated from a multicategory threshold trait, such as kernel row number, vary depending on the method employed.

\section{MATERIAL AND METHODS}

Crosses were made between divergent inbreds for kernel row number. The lines Geneze 3 (G3), Argentino IV (A4) and Dente de Cravo (DC) were used for this purpose. The G3 inbred belongs to the Geneze Seed Company and shows more than 20 kernel rows under most environmental conditions. The inbreds A4 and DC are accessions from Embrapa's maize germplasm bank and have ears of less than 10 kernel rows under environmental conditions. The crosses between the inbred to obtain $\mathrm{F}_{1}$ and $\mathrm{F}_{2}$ and backcrosses $\mathrm{BC}_{11}$ and $\mathrm{BC}_{21}$ were performed at the experimental site of the Federal University of Lavras in August 2008 and Janu- 
ary 2009. The crosses were made between combinations G3 x A4 and G3 x DC.

Two seed populations, $\mathrm{F}_{1}$ and $\mathrm{F}_{2}$, and two backcrosses, $\mathrm{BC}_{11}$ and $\mathrm{BC}_{21}$, were studied at the experimental site of the Federal University of Lavras. The experimental design was a randomized complete block with two replications. The plots of the $F_{1}$ generation were a $2 \mathrm{~m}$ long, with 10 plants per replicate. The $\mathrm{F}_{2}$ generation had 10 rows of $2 \mathrm{~m}$, or 100 plants per replicate and the backcrosses were evaluated in plots of 5 rows of $2 \mathrm{~m}$ for a total of 50 plants. The spacing used between plants was 20 and $90 \mathrm{~cm}$ between rows. Appropriate crop management was carried out in order to provide the best conditions for the plants' development. At physiological maturity, the ears were harvested and husked manually. The number of kernel rows was determined in the harvested ears that showed no failures or pollination problems.

From the data of the averages and average variance within plots, obtained from the analysis of variance, the genetic component means were estimated, in an additive-dominant genetic model without epistasis ( $m, a$ and $d$ ), where $m$ is the midpoint between the homozygotes, $a$ is the bias of homozygous to the average, and $d$ is deviation of the heterozygous. The estimates were obtained by a weighted least squares estimator, as described by Cruz et al. (2001). The components of the phenotypic variance, environmental variance $\left(V_{E}\right)$, additive genetic variance $\left(V_{A}\right)$ and dominance genetic variance $\left(V_{D}\right)$, were estimated by iterative weighted least squares as with the component means, but the weighting used was that proposed by Kearsey and Pooni (1996). We also obtained the narrow sense heritability $\left(h^{2}\right)$ and associated errors according to Rangel et al. (2000).

As this is a threshold trait, the variance components of kernel row number were also estimated by the method proposed by Wright $(1934 a, b)$ and detailed by Falconer and Mackay (1995). By involving more than two thresholds, one gets more than an estimate of the standard deviation of each population. Thus, the phenotypic variance of each population was estimated by the square of the average number of estimated standard deviations of each population. The variance components were obtained algebraically from the expected of the phenotypic variance in the different generations.

\section{RESULTS}

Table 1 shows that the mean of the $\mathrm{F}_{1}$ generation was similar to that of the $\mathrm{F}_{2}$ generation for the character studied, indicating, in principle, that the genes involved should provide additive allelic interaction. Estimates of the variances are consistent with the expected values, since they were higher in the $\mathrm{F}_{2}$ generation. The frequency distribution of $\mathrm{F}_{2}$ is shown in Figure 1. In Figure 1 , one can see large fluctuations, a condition necessary for such a study. The number of rows in the G3 x A4 cross ranged from 12 to 26, while in the G3 x DC cross it ranged from 12 to 28 .

Table 1. Number of observations (No.), means $(\bar{x})$ and variances $\left(\sigma^{2}\right)$ in the crosses evaluated: Geneze $3(\mathrm{G} 3) \mathrm{x}$ Argentino IV (A4) and Geneze 3 (G3) x Dente de Cravo (DC).

\begin{tabular}{lrrrrrrr}
\hline & \multicolumn{3}{c}{ G3 x A4 } & & \multicolumn{3}{c}{ G3 x DC } \\
\cline { 2 - 4 } \cline { 6 - 7 } & No. & $\bar{x}$ & $\sigma^{2}$ & & No. & $\bar{x}$ & $\sigma^{2}$ \\
\hline $\mathrm{F}_{1}$ & 14 & 16.6 & 2.5 & 13 & 18.2 & 5.5 \\
$\mathrm{~F}_{2}$ & 137 & 17.1 & 10.2 & 155 & 19.1 & 9.7 \\
$\mathrm{BC}_{11}$ & 48 & 19.6 & 6.1 & 51 & 17.5 & 6.9 \\
$\mathrm{BC}_{21}$ & 105 & 15.6 & 5.3 & 87 & 17.7 & 3.4 \\
\hline
\end{tabular}



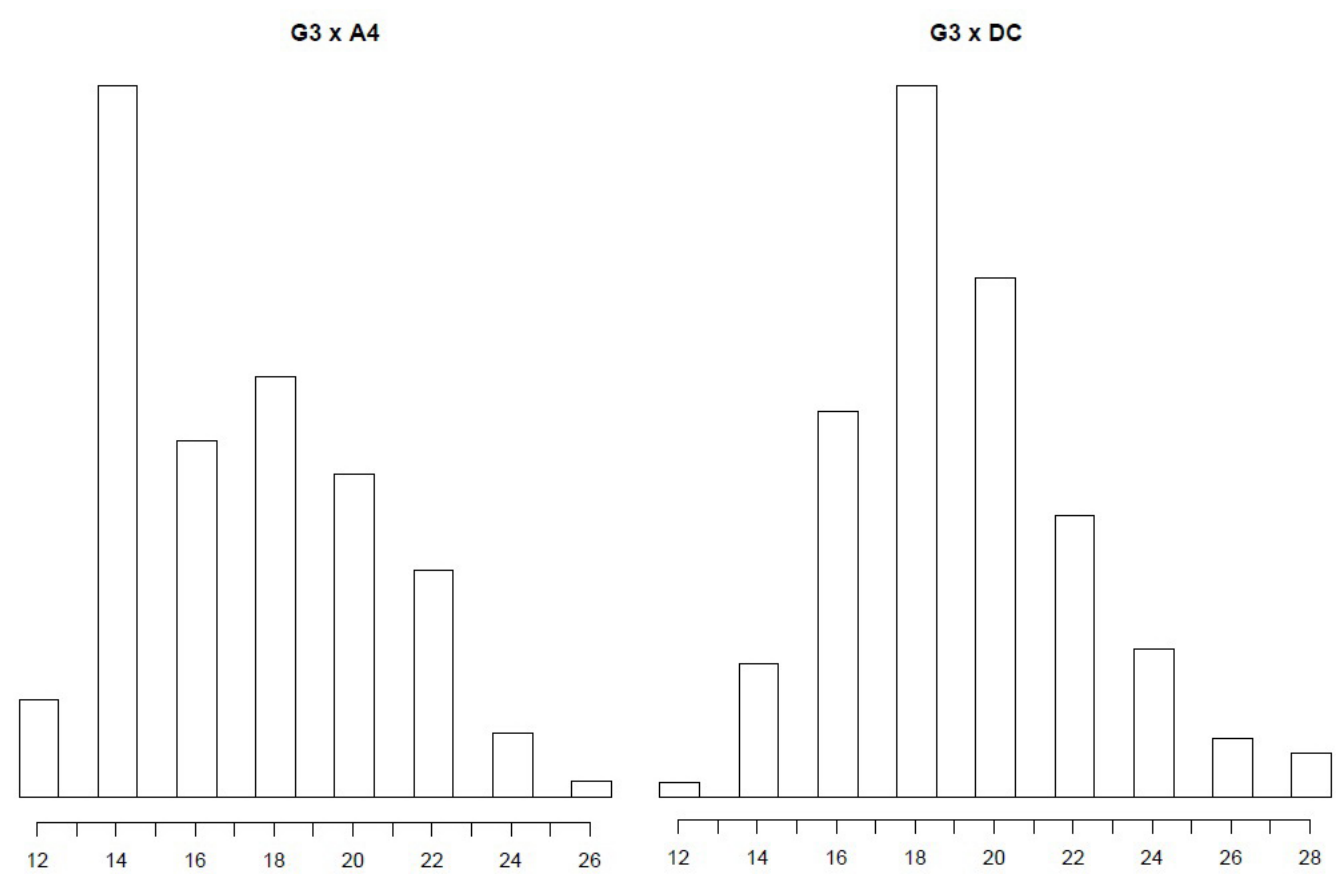

Figure 1. Frequency distribution of the $\mathrm{F}_{2}$ generation for kernel row number in the crosses evaluated: Geneze 3 (G3) x Argentino IV (A4) and Geneze 3 (G3) x Dente de Cravo (DC).

In both crosses, there was an adjustment in the data of the additive dominant model without epistasis, and the coefficient of determination $\left(R^{2}\right)$ of the model was above 0.99 (Table 2 ). It can be seen that the component $a$, which estimates the deviation of homozygotes in relation to the midpoint $m$ between the parents, was greater than zero, which was expected given the magnitude of the difference between the means of parents for the character.

Table 2. Genetic component means ( $m, a$ and $d$ ), associated errors (SE), coefficient of determination $\left(R^{2}\right)$, in the crosses evaluated: Geneze 3 (G3) x Argentino IR (A4) and Geneze 3 (G3) x Dente de Cravo (DC).

\begin{tabular}{lcc}
\hline & G3 x A4 & G3 x DC \\
\hline$m$ & $18.13 \pm 0.45$ & $18.16 \pm 1.89$ \\
$a$ & $3.84 \pm 0.34$ & $0.41 \pm 1.02$ \\
$d$ & $-1.53 \pm 0.76$ & $0.04 \pm 3.5$ \\
$R^{2}$ & 0.999 & 0.999 \\
\hline
\end{tabular}

Data are reported as means $\pm \mathrm{SE}$.

Table 3 presents the estimates of variance components obtained by both methods. It appears that although estimates differ in magnitude, the results are similar in terms of interpretation. This occurred in the two crosses. It should be noted that the additive variance was positive and the variance of dominance did not differ from zero for the two methods. Estimates of heritability for this trait were high. 
Table 3. Components of phenotypic variance, obtained by two methods (LSM and the threshold), associated errors $(s(m))$ and heritability $\left(h^{2}\right)$, in the crosses evaluated: Geneze 3 (G3) x Argentino IV (A4) and Geneze 3 (G3) x Dente de Cravo (DC).

\begin{tabular}{lccrr}
\hline & \multicolumn{2}{c}{ G3 x A4 } & & G3 x DC \\
\cline { 2 - 5 } & LSM & Threshold $^{2}$ & LSM & Threshold $^{2}$ \\
\hline$V_{E}$ & $2.49 \pm 0.32$ & 0.64 & $5.48 \pm 3.14$ & 0.34 \\
$V_{A}$ & $9.45 \pm 0.88$ & 2.18 & $11.68 \pm 3.26$ & 5.61 \\
$V_{D}$ & $-1.75 \pm 0.64$ & -0.02 & $-7.48 \pm 3.72$ & -0.76 \\
$h^{2}$ & $92.72 \pm 19$ & 0.77 & $68.09 \pm 21$ & 0.94 \\
\hline
\end{tabular}

${ }^{1}$ Obtained by the least squares method and reported as means \pm SEM. ${ }^{2}$ Obtained by Wright's method. $V_{E}=$ environmental variance; $V_{A}=$ additive genetic variance; $V_{D}=$ dominance genetic variance.

\section{DISCUSSION}

The parents used were chosen because they showed a wide variation in the number of rows and were not included in the analysis due to low seed germination, and thus can cause stands' problems. The inbred G3 excels due to spikes with 20 or more rows under most environmental conditions. On the other hand, DC and A4 have less than 12 rows. In this condition, the inclusion of parents, primarily to estimate the environmental effect, would affect the interpretation of the results.

The determination of the number of rows is defined ontogenetically and early. Thus, it was expected that there was no variation for the trait in the $\mathrm{F}_{1}$ and that the coefficient of genetic determination $\left(h^{2}\right)$ was 1.0 , since the parents are pure for the trait. One likely cause for this is that, even though ontogenetically determined, there is an environmental influence on trait expression. East (1910) noted this influence of the environment: when the upper ear is lost and there are favorable conditions, the second ear will show two additional kernel rows.

As expected, the $\mathrm{F}_{2}$ generation and backcrosses varied widely in the number of rows of grain, a necessary condition for such a study (Figure 1). Analysis of component means showed that the model without epistasis was sufficiently explained by the data obtained (Table 2).

Estimates of component means showed that dominance is not important in the expression of the trait. Studying the genetic control of some traits of maize ear using molecular markers, Ross et al. (2006) found 10 QTL that explained more than $50 \%$ of the variation in the number of rows, most of these QTL with additive effect. Evaluating other traits of the maize plant, Silva et al. (2004) found additive genetic control for all characters, including kernel row number. Srdic et al. (2007) in a diallel using Hayman's methodology found that the additive effect was more important in the expression of kernel row number.

There was consistency between the results of component means and component variance. This is probably because when you consider one locus, $V_{A}$ is obtained by the expression $2 p(1-p)[a+(1-2 p) d]^{2}$, where $p$ is the frequency of the favorable allele in the population. In this case, since the allele frequency $p=1 / 2$, the additive variance corresponds to $1 / 2 a^{2}$. Thus, $a$ in the component mean and $V_{A}$ in the component variance must provide similar information, as occurred. $V_{D}$ corresponds to deviations in the linear regression equation, used to obtain $V_{A}$. Bernardo (2002) comments that as the least squares procedure tends to minimize the deviations from the line of regression, $V_{D}$, by definition, is considered to be the smallest possible variation between the genotypic values. Thus, a priori, $V_{A}$ will always be larger than $V_{D}$ in normal conditions. 
Since the middle of the last century, many studies were performed in order to estimate the variance of components for maize ear traits. Hallauer and Miranda Filho (1981) summarized estimates of variance components, where the ratio of variance was 0.1774 for kernel row number, showing partial dominance in trait expression.

The confidence interval of heritability $\left(h^{2}\right)$ showed overlap of the estimates, allowing us to infer that the estimates did not differ between crosses. The comparison of estimates of $h^{2}$ is difficult due to the unit in which it was measured. In this case, heritability was at the individual level. The average of 18 estimates of $h^{2}$ on the level of plot, reported by Hallauer and Miranda Filho (1981) was 57\%; it is clear that $h^{2}$ values obtained in this study were high, exceeding $60 \%$.

As already noted, the genetic control of kernel row number showed estimates that allow us to infer that this is a polygenic character with predominantly additive allelic interaction. This finding corroborates earlier interpretations (Lynch and Walsh, 1997). Even with phenotypic expression occurring discontinuously (always in pair numbers), because of the range observed in many classes, there is a good approximation of the observations for a normal distribution. This enables the analysis of its inheritance as a quantitative trait that varies continuously. Thus, both analyses showed discontinuity of phenotypic expression, as the least squares method tended to produce similar results, which can be seen in Table 3 .

It is noteworthy that the estimates by the Wright method (1934a,b) were consistently smaller than the estimates with the least squares method. This "shrinkage" may be the result of the fact that the method assumes a normal distribution and all its derivation is based on the properties of a perfect normal distribution, which does not occur.

Inheritance of kernel row number is due predominantly to additive allelic interactions. The analysis of the genetic control of the kernel row number trait, both by the least squares method and the method proposed by Wright showed similar results in terms of interpretation. This correspondence is due to adjusting the data to normal distribution.

\section{ACKNOWLEDGMENTS}

Research supported by CNPq. The authors acknowledge the Geneze Corn Seed Company and Embrapa Maize and Sorghum for the assignment of inbreds used in present study.

\section{REFERENCES}

Bernardo R (2002). Breeding for Quantitative Traits in Plants. 1st edn. Stemma Press, Hardbound.

Cruz CD, Regazzi AJ and Carneiro PCS (2001). Modelos Biométricos Aplicados ao Melhoramento Genético. 2nd edn. Universidade Federal de Viçosa, Viçosa.

East EM (1910). A mendelian interpretation of variation that is apparently continuous. Am. Nat. 44: 65-82.

Falconer DS and Mackay TFC (1995). Introduction to Quantitative Genetics. 4th edn. Longman, Essex.

Frank TE and Hallauer AR (1997). Generation means analysis of the twin-ear trait in maize. J. Hered. 88: 469-474.

Hallauer AR and Miranda Filho JB (1981). Quantitative Genetics in Maize Breeding. Iowa State University, Ames.

Kearsey M and Pooni H (1996). Genetical Analysis of Quantitative Traits. Garland Science, London.

Lynch M and Walsh B (1997). Genetics and Analysis of Quantitative Trait. Sinauer Associates, Stamford.

Rangel PHN, Pereira JA, Morais OP, Guimarães EP, et al. (2000). Genetic gains for grain yield by irrigated rice breeding program in the mid-north region of Brazil. Pesq. Agropec. Bras. 35: 1595-1604.

Ross AJ, Hallauer AR and Lee M (2006). Genetic analysis of traits correlated with maize ear length. Maydica 51: 301-313.

Silva AR, Souza CL, Aguiar AM and Souza AP (2004). Estimates of genetic variance and level of dominance in a tropical maize population. I. Grain yield and plant traits. Maydica 49: 65-71. 
Srdic J, Pajic Z and Drinic-Mladenovic S (2007). Inheritance of maize grain yield components. Maydica 52: 261-264. Wricke G and Weber WE (1986). Quantitative Genetics and Selection in Plant Breeding. De Gruyter, New York.

Wright S (1934a). An analysis of variability in number of digits in an inbred strain of guinea pigs. Genetics 19: 506-536.

Wright S (1934b). The results of crosses between inbred strains of guinea pigs, differing in number of digits. Genetics 19: 537-551.

Wright S (1943). An analysis of local variability of flower color in Linanthus parryae. Genetics 28: 139-156. 